\title{
Le pas suspendu du dessin
}

\section{François Matton}

\section{OpenEdition}

\section{Journals}

Édition électronique

URL : http://journals.openedition.org/genesis/1692

DOI : 10.4000/genesis. 1692

ISSN : 2268-1590

\section{Éditeur :}

Presses universitaires de Paris Sorbonne (PUPS), Société internationale de génétique artistique littéraire et scientifique (SIGALES)

\section{Édition imprimée}

Date de publication : 12 décembre 2016

Pagination : 87-101

ISBN : 9791023105490

ISSN : 1167-5101

\section{Référence électronique}

François Matton, «Le pas suspendu du dessin », Genesis [En ligne], 43 | 2016, mis en ligne le 29 octobre 2017, consulté le 19 avril 2019. URL : http://journals.openedition.org/genesis/1692 ; DOI : 10.4000/genesis.1692

Ce document a été généré automatiquement le 19 avril 2019.

Tous droits réservés 


\title{
Le pas suspendu du dessin
}

\author{
François Matton
}

1 Si je m'efforce de me remémorer mes premiers dessins, ceux que j'ai bien dû faire à cet âge où tous les enfants paraissent des génies du trait, je n'arrive à rien qu'à une sorte de blanc. C'est ennuyeux. J'aurais aimé y voir rétrospectivement l'embryon du travail que j'ai développé par la suite. Mais non, rien à faire, amnésie totale. Mes parents n'ayant pas gardé une seule trace de ces tout premiers dessins, il me faut accepter de n'en savoir probablement jamais rien. Étaient-ils fougueux, rageurs, timides ou calmes? Faisaient-ils preuve d'un imaginaire puissant, tourmenté, obsessionnel ou, au contraire, pacifié et heureux ? Je ne le saurai jamais. Non, décidément, c'est ennuyeux.

2 Les premiers dessins dont je me souviens sont assez tardifs. J'étais sorti de la petite enfance, peut-être même déjà de l'enfance. Il s'agissait essentiellement de copies prenant appui sur deux univers bien distincts où je puisais tour à tour : les grands maitres de la peinture d'un côté (Véronèse, Rubens, Velasquez, Rembrandt, Watteau, Tiepolo, Guardi, etc.), de l'autre les dessinateurs de bandes dessinées que j'aimais le plus (Moebius, Hugo Pratt, Bourgeon, Gillon, Tardi, Brescia...). Ces copies étaient les plus fidèles possibles, sans la moindre fantaisie, sans la moindre tentative de réinterprétation personnelle. Il semble donc qu'il ne s'agissait pour moi que d'entrer dans le secret des peintres et dessinateurs que j'aimais, en vue de développer une maîtrise technique et plastique. Pourtant je pense rétrospectivement qu'il y avait autre chose: plus que le seul désir d'apprendre le «métier ", il y avait également le plaisir de chevaucher différents univers et de jongler avec un grand nombre d'images que je m'appropriais par le seul fait de les recopier. Je ne peux pas dire que c'était conscient, pourtant je crois que c'est à cette époque qu'est née en moi la jubilation de jouer avec les images les plus variées possibles. Je ne ferai pas autre chose, bien des années plus tard, lorsque j'irai à la pêche aux images sur le Net pour puiser de quoi nourrir mes pages.

3 La juxtaposition faussement aléatoire d'images est peut-être la première caractéristique de mon travail. 


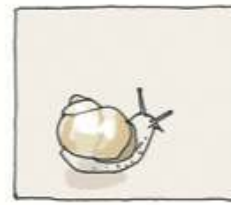

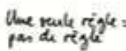

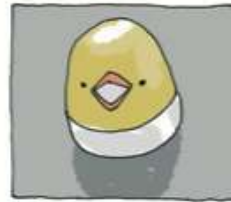

un accueil amurie

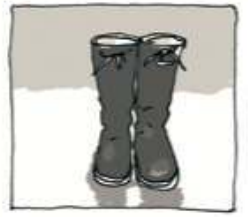

sones excis

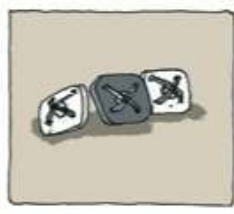

jinste an pen dittention

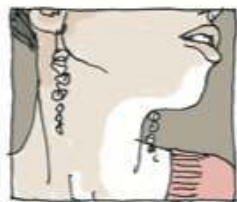

uncetendresc fidice

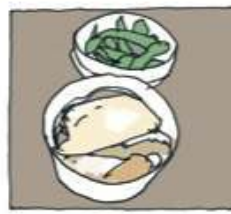

sons penvée

doc 0.tif

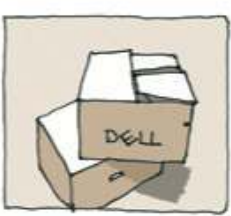

inte un pen de calme

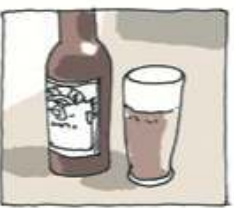

Lo sens aiguikés

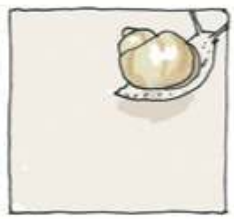

seans mitron

Fig. 1 : Planche extraite de Sous tes yeux (P.O.L/La Cinquième Couche, 2008), représentative de ce principe de juxtaposition apparemment aléatoires d'images

Ce n'est pas qu'une façon de procéder, cela déborde ma petite cuisine personnelle. Il s'agit de ce qui constitue la visée poétique de mes livres. Il faut que je m'explique sur ce point pour ne pas paraitre ronflant. Je n'ai jamais eu le souci de la poésie comme genre auquel m'identifier. Par contre, j'ai très tôt senti que je n'avais rien à dire, ou plus exactement que je ne voulais rien dire, rien raconter, ne développer aucun récit. Pourquoi ? Je ne sais pas trop. Peut-être parce que j'ai un imaginaire très pauvre. Et une très mauvaise mémoire. Je ne me souviens pas de mes rêves, mes rêveries ne développent aucun scénario. D'ailleurs, influencé peut-être à l'école des beaux-arts par les professeurs prônant l'art conceptuel contre le métier traditionnel, la notion de « vécu » m'a vite paru suspecte (en tout cas "anti-moderne»), et je n'ai jamais pensé qu'il m'arriverait la moindre chose exceptionnelle sur le plan de l'histoire personnelle. Même à titre de lecteur, les histoires linéaires me tombent vite des mains. Je préfère de loin les textes en fragments (journaux, correspondances, aphorismes, poésie, textes éclatés). Et lorsque je louchais vers la bande dessinée, mon plus grand plaisir était de lire, plutôt que des albums entiers, des revues « à propos » de la bande dessinée - revues qui montraient des extraits où les vignettes se juxtaposaient, formant un délectable coq-à-l'âne plein d'ellipses.

J'ai commencé à mettre en place cette esthétique du fragment à ma sortie de l'école des beaux-arts. Je m'en souviens pour le coup précisément. J'avais parmi mes affaires tout un stock de bandes bristol (chutes restantes de grandes pages découpées). Ne me décidant pas à les jeter, je me suis amusé à y dessiner au crayon tout ce que j'observais dans mon appartement: principalement des objets très ordinaires (assiettes, verres, chaussures, savon, fourchettes, taille-crayons, réfrigérateur, ampoules), mais aussi des dessins réalisés à partir d'images diverses tirées de mes lectures en cours - dessins auxquels s'ajoutaient quelques croquis de mon amie d'alors (portraits et nus). Tout naturellement, 
j'ai commencé à glisser sous ces dessins des bribes de phrases. Cela à vite pris l'allure d'une sorte de journal intime. Je ne savais pas dans quelle direction je m'avançais, et c'est ce qui me plaisait.
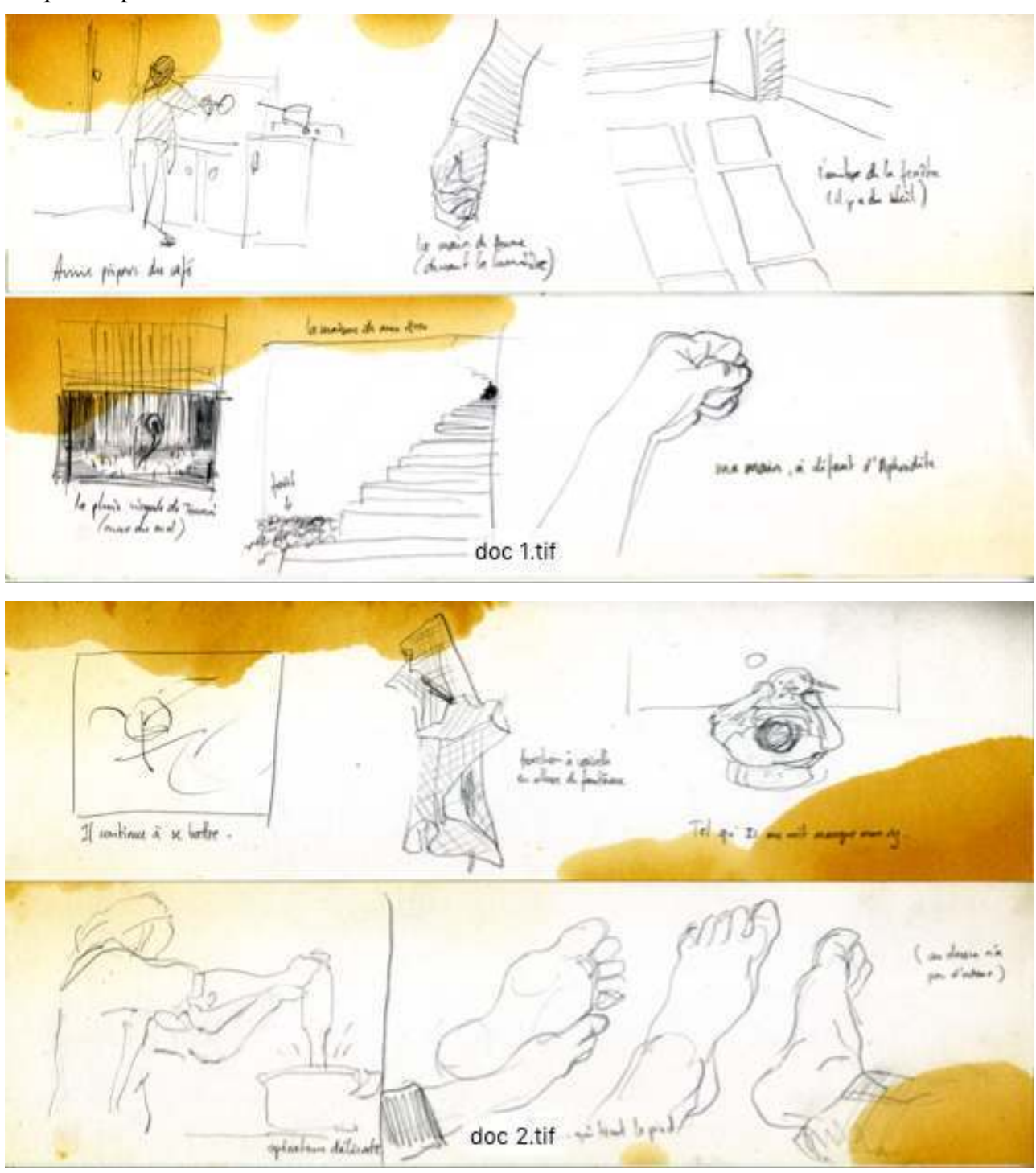

Fig. 2 et 3 : Dessins sur bandes bristols, crayon et huile (archives personnelles, 1993)

6 On pourrait dire que ces bandes de bristol dessinées étaient très littéralement de la «bande dessinée » puisque, à la différence de simples dessins muets, elles se lisaient bel et bien - et de gauche à droite. Toutefois, à la différence des BD classiques, on n'y rencontrait aucun personnage, on n'y voyait aucune bulle. D'ailleurs, je trouvais bien plus naturel de dessiner directement ce que je voyais de mon point de vue. Me représenter comme un personnage m'aurait paru artificiel et passablement naïf. J'évoquais ma vie (parfois dans le détail) au plus près de la manière fragmentaire, elliptique et digressive dont je la vivais. J'avais trouvé là très spontanément une forme originale, très libre, qui me satisfaisait pleinement. Je ne m'en suis guère éloigné par la suite.

7 J'ai repris ce principe d'une seule bande dessinée s'étendant de pages en pages (au format à l'italienne) dans mon premier livre, Lignes de fuites. Mais les dessins sont cette fois circonscrits dans des cases se succédant au nombre de trois par page, le texte se plaçant au-dessous d'elles. 


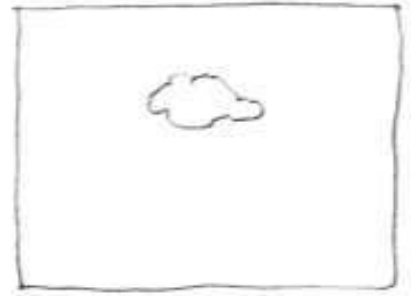

cost dicidí
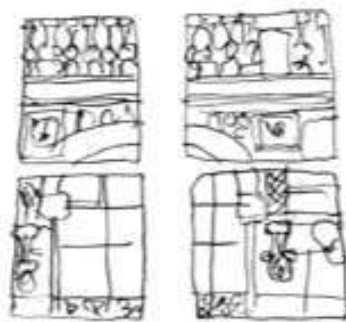

it ${\text { doc } 3 . \text { tif }^{\mathrm{t}}}^{\mathrm{t}}$

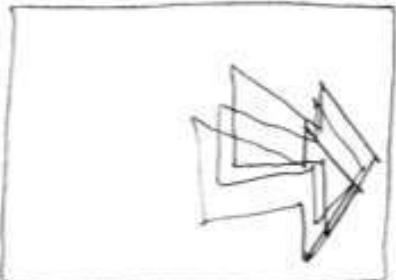

the pax whe nuive

Fig. 4 : Extrait de Lignes de fuites (Dumerchez, 1999) Charlie Brown), mais là encore la référence à la bande dessinée ne m’apparaissait pas essentielle : ce que je voulais avec cette succession de trois cases, c'était trouver la forme minimale et suffisante pour poser une situation basique, comme la construction élémentaire d'une phrase (sujet-verbe-complément). Ma référence louchait donc plutôt du côté de la poésie, et plus précisément du côté du haïku (on sait que la particularité du haïku à saisir l'instant est intimement liée à la forme tripartite : trois vers, trois segments calligraphiés sur trois lignes verticales, un instant suggéré par trois aspects intrinsèquement liés).

Cette forme idéalement équilibrée possède l'avantage de donner naissance à des pages autonomes. Malgré tout je m'arrangeais, en ménageant entre elles des passerelles, pour qu'on puisse également les lire à la suite les unes des autres, comme un tout. Plus tard, deux livres publiés aux éditions P.O.L (J'ai tout mon temps, 2004, et Autant la mer, 2009) reprendront exactement cette même forme - pour moi la forme idéale, la plus proche de ma pensée rêveuse et divagante, la plus à même d'enchaîner en douceur les éléments les plus variés : les choses qui se tiennent dans l'instant sous mes yeux, aussi bien que les images évoquant un ailleurs nomade propice à la rêverie.

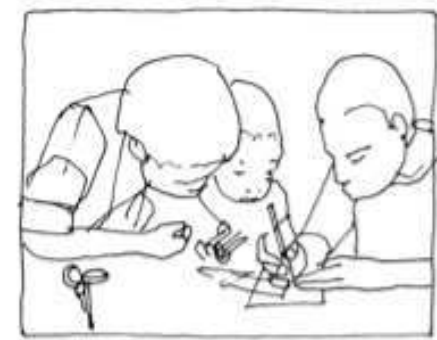

is mis encort tout petit

mon conkin me moutre a quit nait thire en lesinant

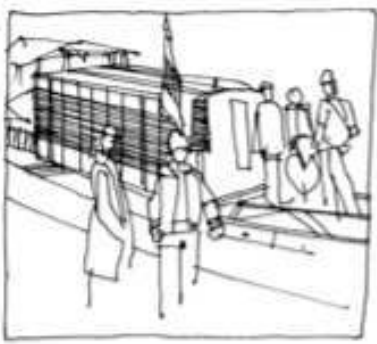

je min ridiré

doc 4.tif

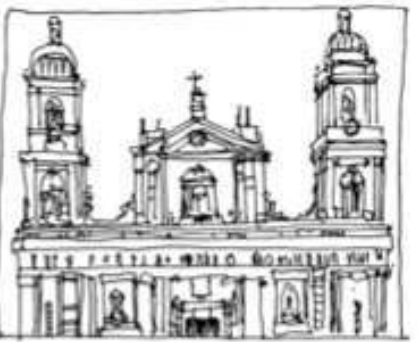

mox counin nit un gémie

Fig. 5 : Extrait de J'ai tout mon temps (P.O.L, 2004) 


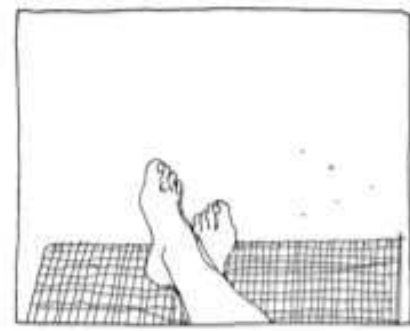

is miritanda encalesom

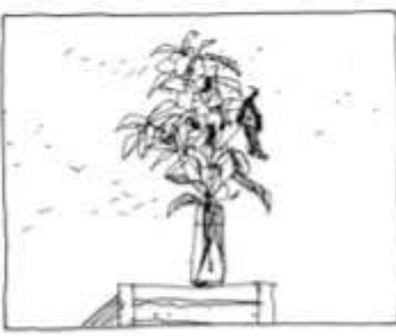

(c) riat gas mive de bonges

doc 5.tif

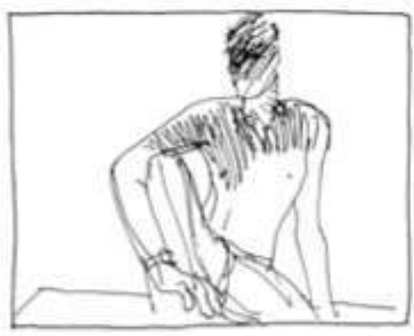

ic mitre le temp de vive

Fig. 6 : Extrait de Autant la mer (P.O.L, 2009)

Sur cet aspect de l'ailleurs, je dois dire que j'ai beaucoup dessiné en feuilletant des revues de voyages accumulées en hautes piles dans le grenier de la maison familiale. Ces magazines (je pense notamment à Grands Reportages) étaient abondamment illustrés. En les feuilletant, alors que j'étais encore adolescent, j'avais à peu de frais l'impression de voyager, de pressentir le frisson de l'aventure - émois érotiques compris. Finalement, un jour, j'ai découpé aux ciseaux toutes les images qui me parlaient pour une raison ou pour une autre. J'ai ainsi constitué une petite banque d'images où j'allais puiser, et cela bien avant qu'Internet n'offre de le faire avec la facilité que l'on sait. En détachant ces photos des légendes et textes qui les accompagnaient, je perdais volontairement leurs références en vue de m'autoriser une plus grande liberté dans la réappropriation que j'allais en faire ensuite.
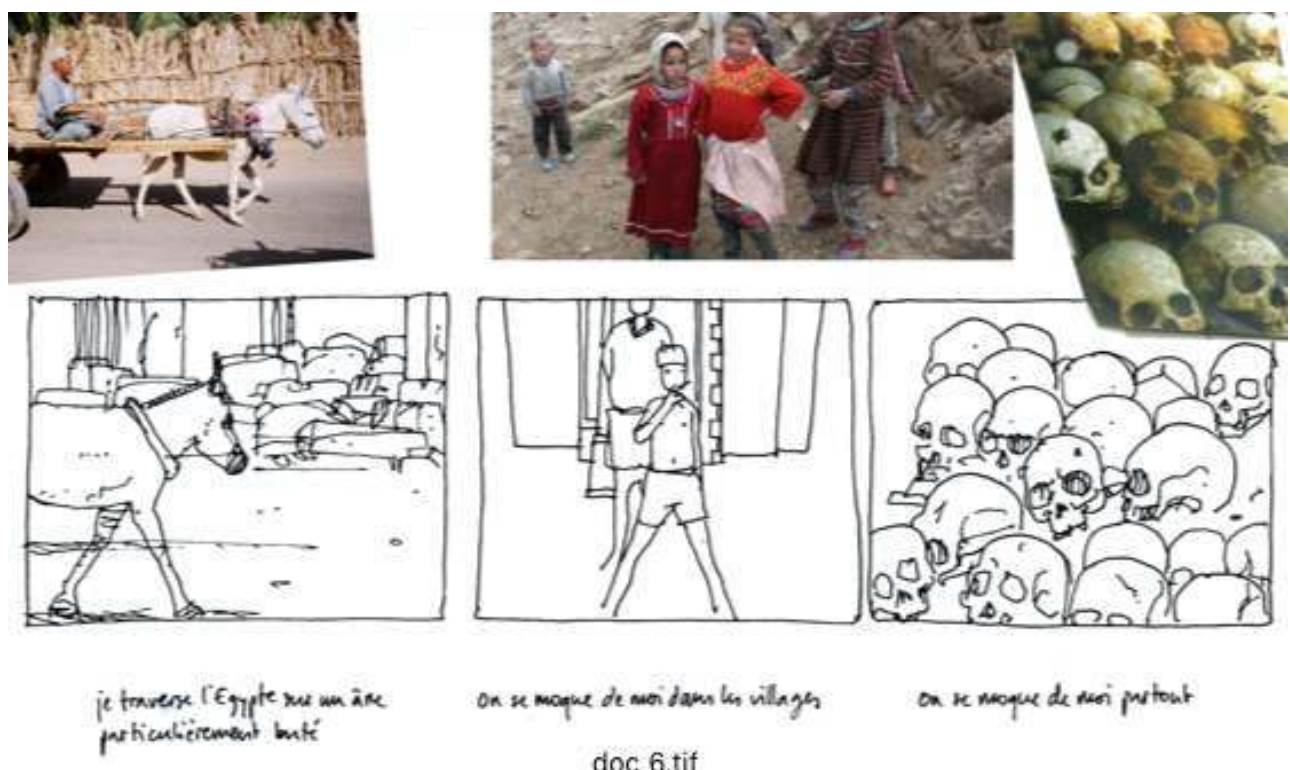

$$
\text { on se moque de nei dans len villages }
$$$$
\text { on renoque de noon pustont }
$$

doc 6. tif

Fig. 7 : Photos extraites de revues de voyage sur lesquelles je me suis basé pour ce strip extrait de J'ai tout mon temps (P.O.L, 2004)

Ainsi, dans mes livres, la juxtaposition d'images a toujours précédé l'écriture. Ce n'est qu'à partir d'une sorte de montage d'images hétérogènes que l'envie me prend d'écrire quelque chose en inventant entre elles un lien. C'est pour moi la plus sûre manière d'activer la rêverie. Mon naturel contemplatif y trouve son compte, interrogeant ces images comme on s'interroge en observant des intérieurs d'appartements entrevus le soir 
à la tombée de la nuit : «Qu'est-ce que ce serait de vivre ici ? Quelle vie m'offrirait un tel décorum?»(fig. 8).

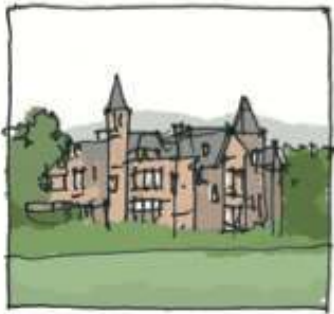

Qu'st-ce quece vernit de vivre ici?

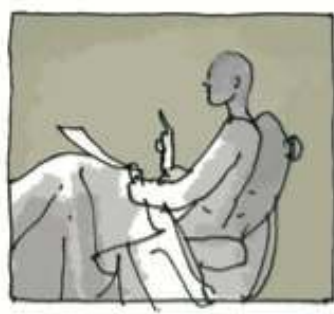

portes par les rieiden

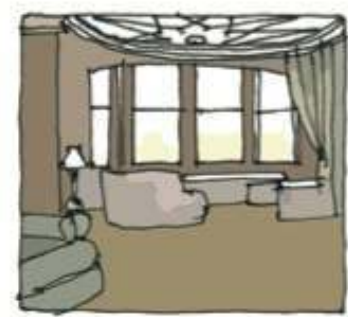

avec l'odeur du pare qui monte resqu' aux bolon 'et insqu' cux chambres

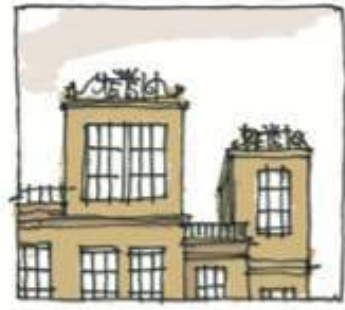

let'imagive studiease Lecrice de gandes temêtres

doc 7.TIF

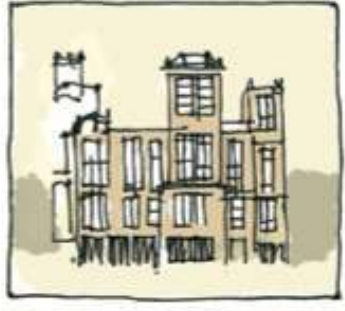

on bienli

a $x$ laiser glimer mar le priquets

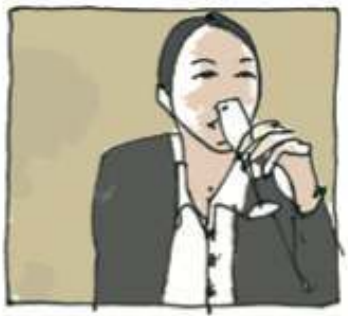

studicuse et gaic le rat verue

Fig. 8 : Un exemple de ce type de rêverie dans cette planche extraite de Sous tes yeux (P.O.L/La Cinquième Couche, 2008)

12 Je procède de la même manière devant chaque image glanée ici ou là. Cette rêverie propice à un certain lyrisme mélancolique conduit rapidement à une appropriation de l'image, au point que je finis par confondre ces images avec les photos que j'ai réellement prises, constituant ainsi de faux souvenirs s'intégrant à ma mémoire comme les vrais. De la même façon, j'aime confondre les dessins que j'ai réalisés en observation directe (dans la tradition du carnet de bord) avec les dessins réalisés d'après des photos dont je ne sais rien (fig. 9). 


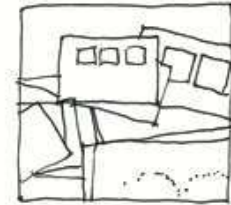

Tout

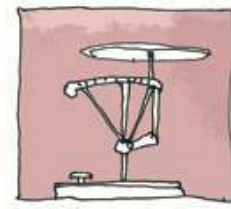

c'st tribs simple

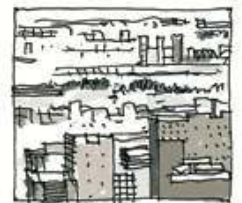

lache tout

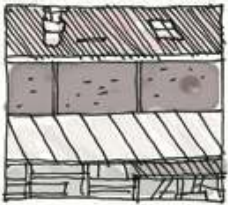

fout ist k

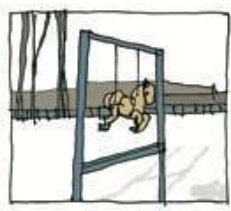

un jendientant

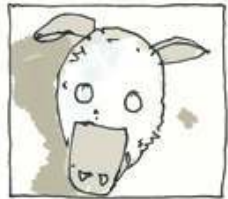

nisie pas peur

doc 8.tif

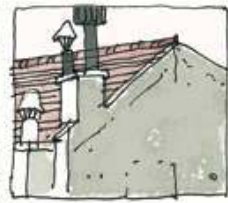

dans timstant

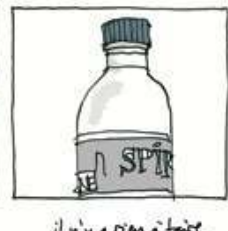

iln'y a rimaitrive

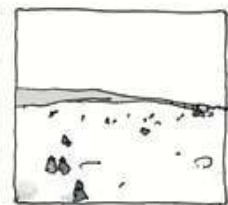

levide vativalear

Fig. 9 : C'est le cas dans cette autre planche extraite de Sous tes yeux (P.O.L/La Cinquième Couche, 2008)

13 C'est une façon de brouiller et d'élargir les frontières de mon intériorité. J'engendre des images « personnelles » à partir d'images « impersonnelles» (je mets des guillemets car pour moi, dans le cadre de ce travail, ces concepts ne sont pas pertinents). J'en arrive à créer une identité poreuse, aux bords incertains, à même de tout intégrer.

Toujours à propos de cette formation des dessins par capillarité, un autre aspect de ma façon de procéder consiste à accumuler en désordre des dessins très divers sur de grandes pages. J'en recadre ensuite des fragments qui seront autant de vignettes dans mes pages. C'est ce que j'appelle le recadrage fétichiste (on sait que le fétiche c'est le désir même, autrement dit la pulsion scopique qui découpe ce qui émeut dans le champ indifférencié du réel). À l'occasion, je peux me servir d'une petite fenêtre découpée dans une feuille de papier que je fais glisser sur mes dessins pour recadrer les morceaux de choix (fig. 10). 


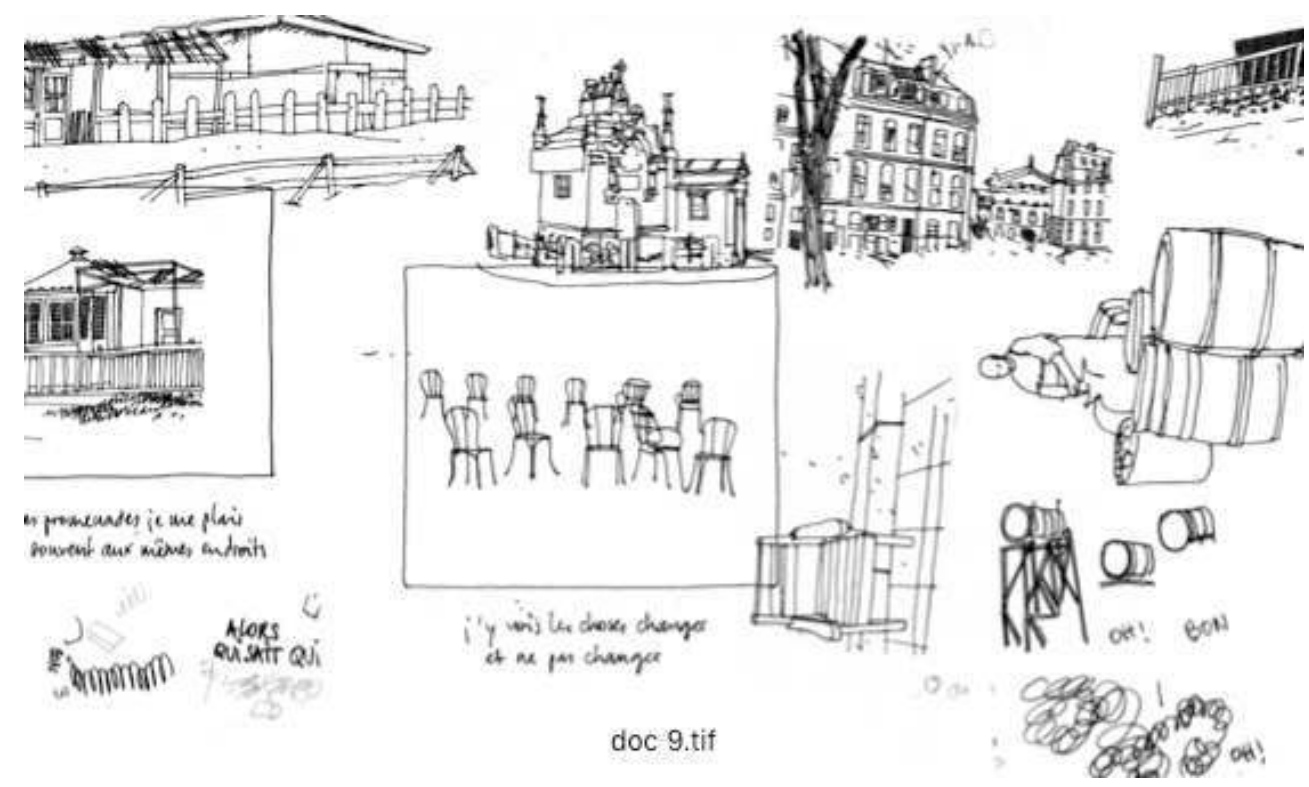

Fig. 10 : Un exemple de cette façon d'opérer (dessin non daté, archive personnelle) bruit et de silence. C'est aborder un aspect psychologique lié à un besoin personnel de vivre en retrait, besoin si puissant qu'il dicte grand nombre de mes choix plastiques. En effet, j'ai toujours eu le sentiment oppressant que nous vivons dans un monde assailli par beaucoup trop d'images et d'informations, je souffre tellement de cette saturation de l'espace qu'il me faut impérativement faire le vide pour respirer. Faire le vide, c'est la tentation de la tabula rasa en vue d'oublier un temps tout ce qui s'est fait, tout l'art et la littérature. Faire le vide pour retourner au point zéro (comme disent les maîtres du zen), pour retrouver l'espace avant la représentation. Se tenir juste là, face à la feuille blanche, et se contenter de l'observer dans sa virginité vivifiante. calme et de retrait. C'est assumer mes limitations personnelles et tenter de faire de ma faiblesse une force - du moins un moteur pour le travail.

"L'aventure commence par le vide » (ainsi que je l'ai écrit dans la préface de Lignes de fuite) : faire table rase donc - littéralement : mettre à nu la table de travail -, pour renouer avec une forme d'insouciance, pour redevenir léger, pour commencer à s'amuser. Condition pour travailler dans la jubilation.

18 Cela se traduit par la case laissée vide dans la page, façon de ménager un espace qui ne s'est pas encore déployé. Un espace à naître, riche de tous les possibles (fig. 11).

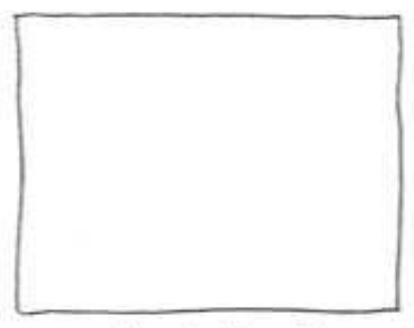

a sorat le iedre dime hitaire

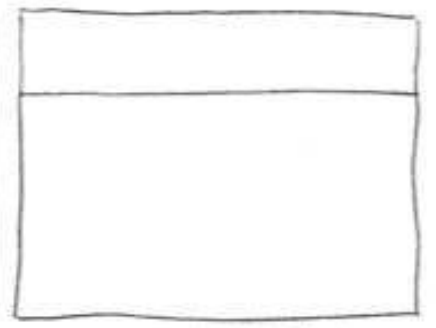

ce xna doc 10.tif hisforic

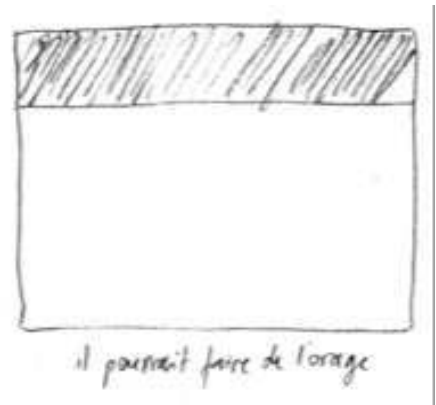

Fig. 11 : Comme on le voit dans cet extrait de Lignes de fuites (Dumerchez, 1999) 
19 pourrait arriver ", comme aux premières secondes de la projection d'un film, avant qu'on entre dans une histoire, avant que des personnages apparaissent. C'est une façon de suggérer que le plus important pourrait être ce temps précieux et fragile où rien n'est encore affirmé.

Quoi qu'il en soit de cette utopie discutable d'un retour au vide se suffisant à lui-même (jusqu'à rendre inutile toute action, notamment artistique), c'est à partir de cette considération du vide, et après m'être placé dans un retrait vigilant, que je peux établir avec le monde un rapport amoureux. Ce n'est qu'à partir du vide que je peux m'amuser à me projeter. Façon de ne pas faire partir le travail de moi, de ma petite histoire, de mon petit imaginaire, de mes petites souffrances et conflits personnels ( $c f$. Deleuze reprochant à la littérature française d'être issue la plupart du temps d'un désir "de régler ses comptes avec papa-maman » et plombée par «le sale petit souffle de l'intériorité », en opposition à la littérature américaine qu'il voit toute entière ouverte sur « le vent frais du dehors $\left.»^{1}\right)$.

C'est dans cet esprit que j'ai écrit Sous tes yeux, soit un livre conçu comme une invitation faite au lecteur de tourner son attention vers le réel immédiat et la présence ordinaire des choses infiniment variées qui nous entourent. C'est à mes yeux ce que le dessin peut nous apporter de plus précieux, bien plus précieux que de raconter une histoire de plus, où les personnages masquent bien souvent la présence matérielle du monde (du moins la faisant passer à l'arrière-plan) : nous faire aimer non seulement les personnes, mais aussi (et surtout) les arbres, les nuages, tous les objets qui nous entourent - tasses, lit, armoire, rouleau de scotch. Cette grande variété de choses et d'objets est montrée sans hiérarchie dans le livre, tout y est placé au même niveau, la présence humaine n'occupe pas le premier plan. Ce statut des choses représentées rejoint l'absence de catégories discriminantes propre à la vision du bouddhisme zen. Le regard se désencombre de toutes les histoires et de toutes les projections qui s'interposent entre nous et les choses. Le monde devient un spectacle permanent, aucun divertissement n'est plus nécessaire, ouvrir les yeux suffit à nous combler. 


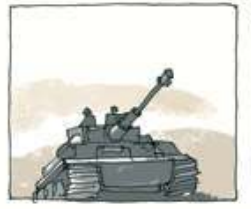

matingas

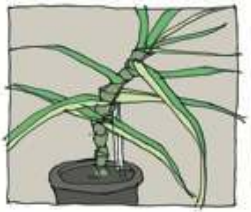

tiger inconfort

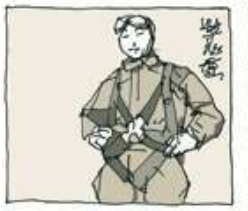

qual cinima!

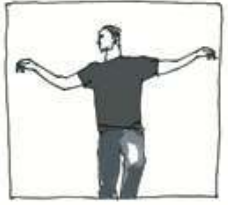

quelques youttes de pluie

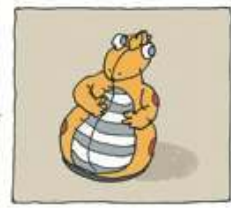

Letike proche

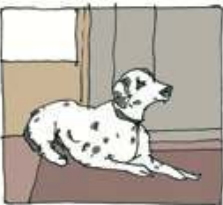

allez, oust!

doc 11.tif

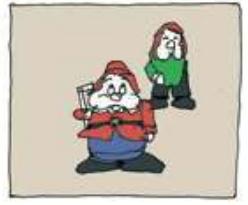

sempirs

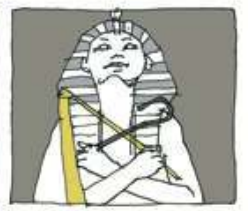

vellkitt's dim autre àge

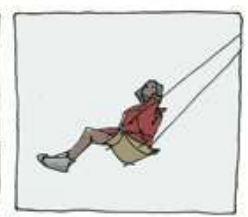

tout le monke dechors!

Fig. 12 : Extrait de Sous tes yeux (P.O.L/La Cinquième Couche, 2008)

On ne s'étonnera pas après avoir lu ce credo que mon dernier livre en date ait pour titre 220 Satoris mortels (P.O.L, 2013). Cette fois, afin de pointer directement vers l'expérience de basculement de la conscience propre au satori, les dessins ne sont plus circonscrits dans une case, et chaque page n'en montre qu'un, central. Les petits textes/poèmes qui les accompagnent sont disposés librement autour du dessin (en haut, en bas ou sur le côté). Je m'éloigne on ne peut plus de l'aspect séquentiel de la bande dessinée (fig. 13). 


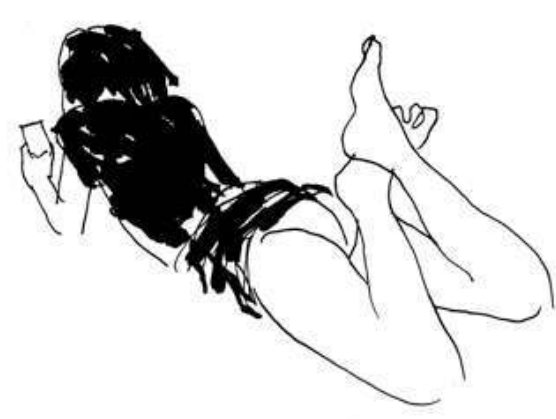

doc 12. tif

Fig. 13 : Page extraite de 220 Satoris mortels (P.O.L, 2013)

Tout récit linéaire (horizontal) est abandonné au profit d'une lecture de nature poétique (verticale). Pas de personnage identifiable, pas de narrateur, pas de récit linéaire. Et pourtant: des visages, des corps, des situations, des moments de crise, du désir, et même du suspens (que se passe-t-il au juste? chance ou malchance? dans quel sens tourne le vent? comment cela va-t-il se finir?). Des instants se succèdent, qui sont autant de basculements dans l'espace indistinct qui se tient à l'arrière-plan de toute histoire. Pour cela le dessin (central) forme une clé au même titre que le texte (minimal). De l'un à l'autre, du dessin au texte et du texte au dessin, ce qui se joue, loin de tout rapport d'illustration ou de redondance, est la possibilité de lier toujours différemment ce qui n'a initialement pas vocation à l'être. Ainsi, la plupart du temps, la légende s'inscrit en décalage avec le dessin et l'histoire reste à inventer. L'initiative en est laissée au lecteur. Ce rébus ou cette énigme lui est destiné. 
QUAND UN SOWENIR ÉROTIQUE

TRES PRECIS

SE RÉVEIUE EN PLENN SOMMEIL

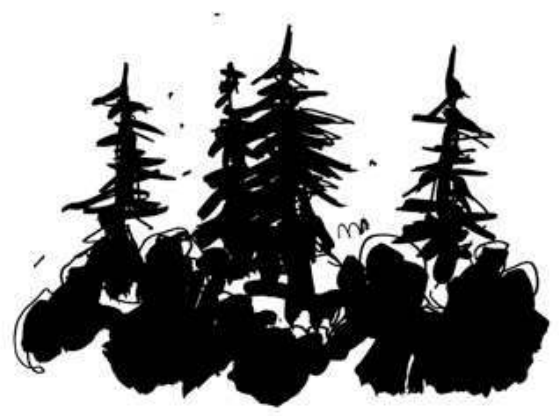

varo mines?

doc 13.tif

Fig. 14 : Page extraite de 220 Satoris mortels (P.O.L, 2013)

Parallèlement à la réalisation de ces livres, je publie régulièrement, depuis 2006, sur mon «blog à dessin» (http://francois-matton.blogspot.fr/), sorte d'atelier en ligne conçu comme un laboratoire pour expérimenter les liens les plus divers entre textes et dessins (fig. 15). Les expériences narratives ou poétiques esquissées sur ce blog se rapprochent parfois des expériences à contraintes de l'Oubapo (l'Ouvroir de bande dessinée potentielle fondé en 1992 par la maison d'édition L'Association). Souvent déconcertantes, elles se dressent toutes contre la notion d'illustration redondante. 


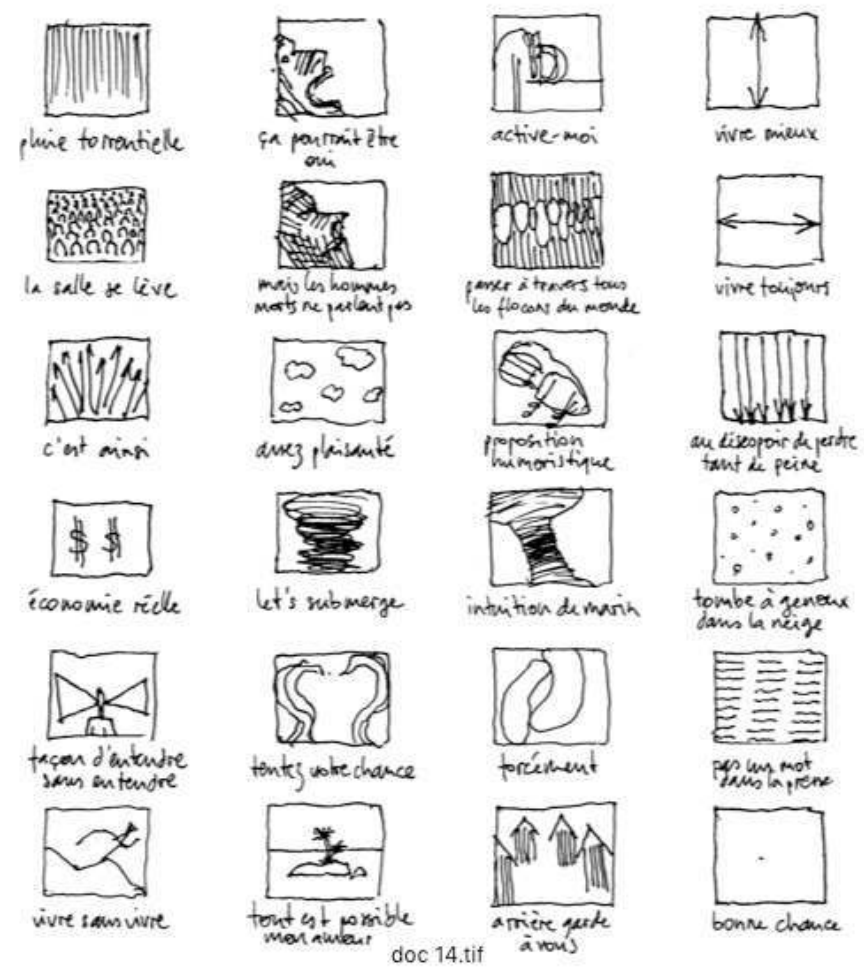

Fig. 15 : Dessins extraits du blog (sans date)

Il est toujours possible d'inventer de nouveaux dispositifs formels (non formalistes) pour sortir des habitudes de lecture plus ou moins lénifiantes auxquelles conduit une pratique de la bande dessinée qui ne remettrait pas en cause le lien illustratif entre dessin et texte. L'un de ces dispositifs consiste à s'appuyer sur des sujets différents de ceux que la mauvaise littérature et la médiocre bande dessinée drainent depuis toujours. Par exemple, en louchant du côté des sciences (la botanique, la zoologie, l'astronomie, l'archéologie, les sciences sociales, etc.), dont on s'amusera à emprunter la méthode et les tournures pour les parodier. De même, il peut être intéressant d'emprunter à la philosophie ou à la spiritualité certaines approches spécifiques du monde, en jouant à mêler les niveaux de discours. En diversifiant ainsi les sources iconiques, on se retrouve à travailler par montage, à la manière des cinéastes. On sait qu'avec le montage d'images, même sans texte, on est déjà dans la narration. Lorsque ces images montées sont disjonctives, la narration qui en découle est particulièrement ouverte à de multiples interprétations. Le sens circule librement, il n'est pas posé de façon univoque. Le lecteur doit faire l'effort de le découvrir ou de l'inventer, ce qui permet d'établir avec l'œuvre un lien fort et vivant basé sur l'ambivalence du jeu - source de jubilation.

Certaines de ces expériences menées librement dans l'espace du blog pourront ouvrir des pistes pour de futurs projets. C'est précisément le cas pour mon dernier livre paru, Oreilles Rouges et son maitre (P.O.L, 2016), dont les premiers épisodes ont vu le jour sur mon blog en 2013. 


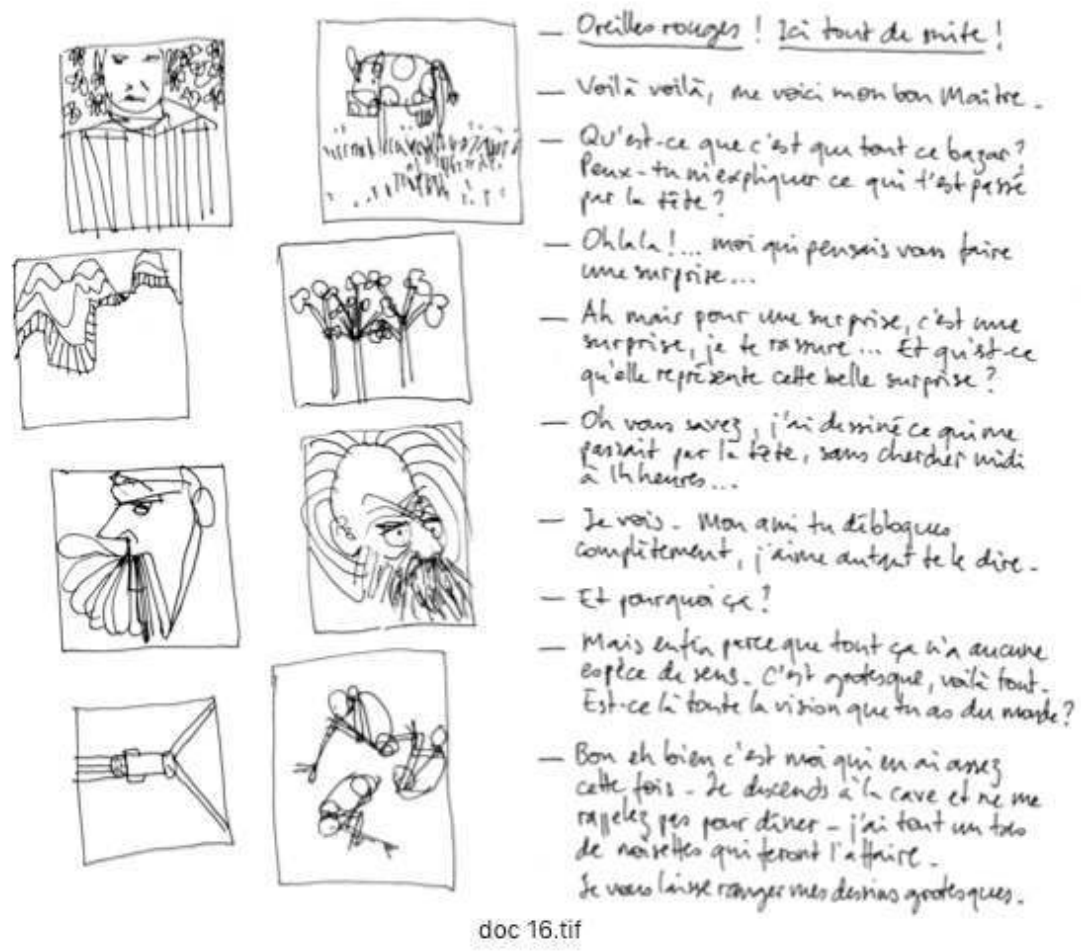

OREILLES ROUGES SE FÂCHE

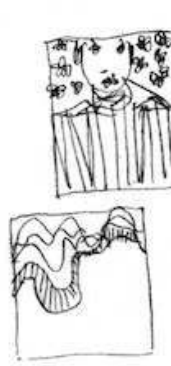

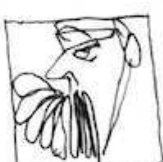
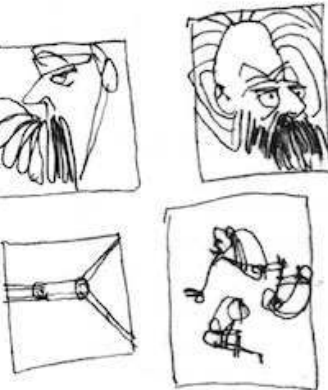

- Oreilles Rouges! Ici tout de suite!

- Voilà voilà, me voici, mon bon Maitre.

- Qu'est-ce que c'est que tout ce bazar? Peux-tu me dire ce qui t'a pris?

- Oh là là!... Moi qui pensais vous faire une surprise...

- Ah mais, pour une surprise, c'est une surprise, je te rassure. Et qu'est-ce qu'elle représente cette belle surprise?

17

Fig. 16 et 17 : Un épisode de Oreilles Rouges et son maître extrait du blog à dessin et son état final publié 


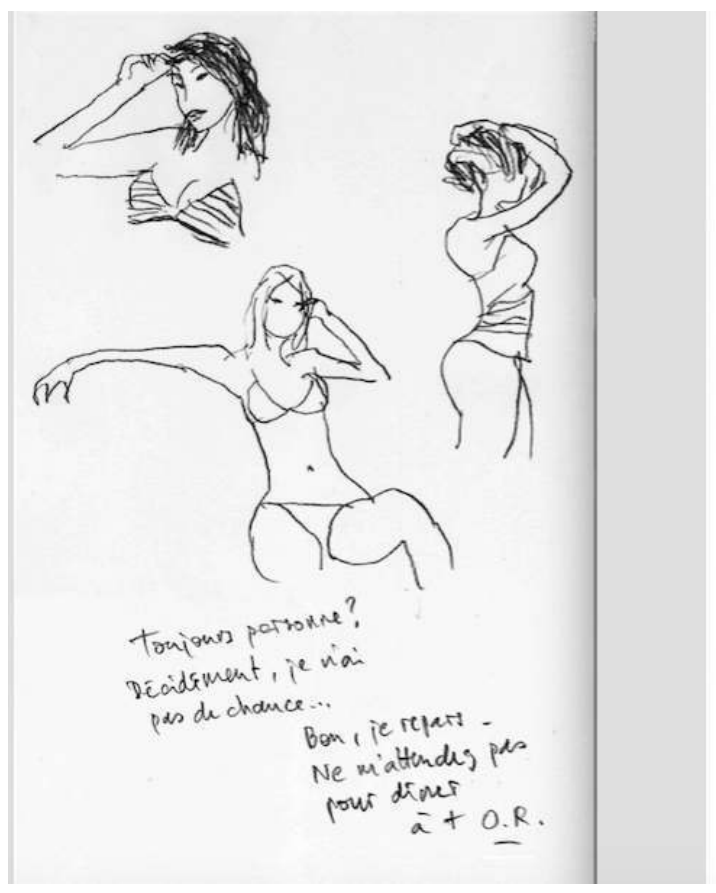

42

OREILLES ROUGES ENFONCE LE CLOU

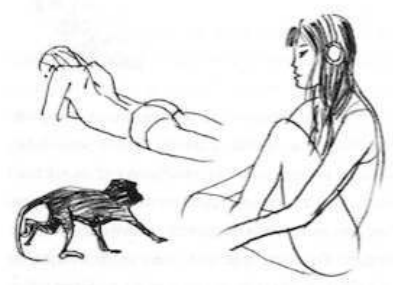

Toujours personne? Décidément, je n'ai pas de chance. J'étais repassé pour

128

Fig. 18 et 19 : Un épisode de Oreilles Rouges et son maître extrait du blog à dessin et son état final publié

\section{NOTES}

1. Dans Dialogues, avec Claire Parnet, Paris, Flammarion, coll. » Champs », 1996.

\section{RÉSUMÉS}

François Matton, auteur de livres publiés par les éditions P.O.L, expose, dans un texte aux allures d'enquête autobiographique, la génétique de ses ouvrages où textes et dessins tissent des rapports très singuliers et constamment renouvelés, jusqu'à constituer une œuvre aux innombrables expérimentations formelles ouvrant sur des pistes narratives relevant tout à la fois du journal intime, de la philosophie et du haïku néo-Beat. Le dessin se présente alors comme une occasion d'étendre à l'écriture les signes de la main en vue de pointer vers un singulier rapport au monde embrassé dans toutes ses dimensions.

In a text that recalls an autobiographic investigation, François Matton, the author of books published by P.O.L, presents the genesis of his works where texts and drawing are interwoven in highly singular and constantly renewed relationships, forming a work with countless formal 
experiments leading to narrative paths that associate the diary, philosophy and neo-Beat haiku. In this case the drawing appears to be an opportunity to extend to writing the signs of the hand, seeking a singular relation with the world envisaged in all its dimensions.

François Matton, cuyos libros son publicados por la editorial P.O.L, expone -en un texto que se aproxima a la investigación autobiográfica- la genética de sus obras, en las cuales textos y dibujos entretejen vínculos singulares y permanentemente renovados, hasta constituir una obra con innumerables experimentaciones formales, que abren pistas narrativas que dan cuenta, al mismo tiempo, del diario íntimo, de la filosofía y del haiku neo-beat. El dibujo se presenta, en esta perspectiva, como una ocasión para extender hasta la escritura los signos de la mano, apuntando a una relación singular con el mundo, abarcado en todas sus dimensiones.

François Matton, autore di libri pubblicati dalle edizioni P.O.L, espone, in un testo che ha le caratteristiche di un'inchiesta autobiografica, la genetica delle sue opere, dove testi e disegni intessono rapporti molto singolari e costantemente rinnovati, fino a costituire un'opera dalle innumerevoli sperimentazioni formali che si aprono su piste narrative appartenenti, allo stesso tempo, ai domini del diario intimo, della filosofia e dell'haiku neo-Beat. Il disegno si presenta allora come un'occasione di estendere alla scrittura i segni della mano, puntando verso un singolare rapporto con il mondo, abbracciato in tutte le sue dimensioni.

François Matton ist Autor von Büchern, die vom Verlag P.O.L veröffentlicht wurden. In einem Text nach der Art einer autobiographischen Untersuchung legt er die Genese seiner Werke dar, wo Text und Zeichnung sehr einzigartige und beständig erneuerte Verbindungen eingehen, bis zu dem Punkt, ein Werk mit zahllosen formellen Versuchen zu konstituieren, welche neue narrative Wege eröffnen, welche gleichzeitig dem Tagebuch, der Philosophie und dem Neo-BeatHaiku angehören. Die Zeichnung bietet so die Gelegenheit, die Schrift als Zeichen der Hand zu verstehen, um eine einzigartige Beziehung zur Welt aufzuzeigen, welche alle ihre Dimensionen umspannt.

\section{AUTEUR}

\section{FRANÇOIS MATTON}

François Matton, né en 1969, conduit depuis une vingtaine d'années une entreprise très singulière. Venu du monde des beaux-arts, il collabore tout d'abord avec Renaud Camus chez P.O.L puis fait évoluer son dessin vers des dispositifs nouveaux, qui relèvent à la fois de la contrainte (série des cases de même format alignées avec régularité) et de la liberté d'improvisation la plus grande (pleine page, esquisses et brouillons, mélange de textes). La notion de bande dessinée prend ainsi chez lui une dimension très plastique. Elle peut par exemple s'étendre à toute la page, dans des albums-fresques spectaculaires comme De pièces en pièces (P.O.L, 2007), qui fait entrer le regard dans l'espace des lieux d'exposition. Ou bien jouer de l'alternance du texte manuscrit et du dessin sur une surface parfaitement blanche, à l'instar de Comment j'ai cassé mes jouets (PetitPol, 2005). Dans Sous tes yeux (P.O.L et La Cinquième Couche, 2008), c'est une syntaxe et un phrasé de l'image qui apparaissent, avec un jeu de décalage troublant entre le texte et l'image qui fait songer à certaines expérimentations de Jean-Luc Godard. Plus largement, François Matton donne une dimension philosophique à ses textes graphiques. Que ce soit dans J'ai tout mon temps, ou dans Oreilles Rouges et son maître (P.O.L, respectivement 2004 et 2015), la méditation favorise une pensée du regard sur le monde. Son rapport au dessin, le lien étroit entre l'édition finale et l'atelier public du blog, le réalisme méditatif de ses haïkus graphiques constituent un ensemble exceptionnel dans le paysage 
contemporain de la bande dessinée. Pour Genesis, François Matton a accepté de revenir sur ses naissances graphiques successives et sur les processus à l'œuvre dans la forge des albums. 